\title{
Belgeo
}

Revue belge de géographie

$1 \mid 2021$

Miscellaneous

\section{Quartiers anciens dédiés au tourisme et aux loisirs en Chine. Pratiques et représentations des usagers de NanTangLaoJie à Ningbo}

old districts dedicated to tourism and leisure in China. Practices and representations of NanTangLaoJie users in Ningbo

\section{Annie Ouellet}

\section{OpenEdition}

\section{Journals}

Édition électronique

URL : https://journals.openedition.org/belgeo/47375

DOI : 10.4000/belgeo.47375

ISSN : 2294-9135

Éditeur :

National Committee of Geography of Belgium, Société Royale Belge de Géographie

\section{Référence électronique}

Annie Ouellet, «Quartiers anciens dédiés au tourisme et aux loisirs en Chine. Pratiques et

représentations des usagers de NanTangLaoJie à Ningbo », Belgeo [En ligne], 1 | 2021, mis en ligne le 11 mai 2021, consulté le 24 octobre 2021. URL : http://journals.openedition.org/belgeo/47375 ; DOI https://doi.org/10.4000/belgeo.47375

Ce document a été généré automatiquement le 24 octobre 2021.

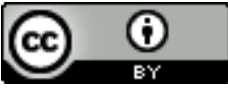

Belgeo est mis à disposition selon les termes de la licence Creative Commons Attribution 4.0 International. 


\title{
Quartiers anciens dédiés au
} tourisme et aux loisirs en Chine. Pratiques et représentations des usagers de NanTangLaoJie à Ningbo

\author{
old districts dedicated to tourism and leisure in China. Practices and \\ representations of NanTangLaojie users in Ningbo
}

Annie Ouellet

\section{Introduction}

1 Dans les années 1960, Joffre Dumazedier défendait la thèse selon laquelle les sociétés occidentales deviendraient une « civilisation du loisir» (Dumazedier, 1972 [1962]). Ces sociétés ont effectivement opéré une transition où l'augmentation de la productivité et les progrès techniques ont permis de faire diminuer le temps de travail et de dégager du temps libre et octroyer une place centrale aux loisirs et au tourisme (Merle, 2017). L'avènement de cette société du loisir, entre autres caractérisée par le fractionnement du temps de travail, contribue à fragiliser la frontière entre tourisme et loisirs. Dans un tel contexte, les mêmes espaces voient se côtoyer touristes, excursionnistes et résidents venant habiter ${ }^{1}$ des lieux plébiscités pour leur caractère agréable, ludique ou historique.

2 Par ailleurs, les sociétés ayant réalisé ou étant en voie de réaliser leur transition dépassent aujourd'hui largement les limites de l'Occident. Certes, les sociétés occidentales ont été les premières à se transformer mais cette civilisation du loisir concerne aujourd'hui des sociétés de plus en plus nombreuses à travers le monde. Presque tous les territoires tentent d'aménager des lieux pour le tourisme et les loisirs et ainsi attirer des visiteurs venus d'horizons plus ou moins lointains. Les attraits créés ou mis en valeur sont variés, allant de complexes récréatifs à des espaces naturels 
préservés ou encore des traces laissées par des civilisations passées, que nous nommerons ici le patrimoine ${ }^{2}$ culturel. Le processus de désignation de ce patrimoine (patrimonialisation), comme celui de mise en tourisme, a connu des transformations importantes depuis ses origines. Les biens désignés en tant que patrimoine sont plus variés (en matière de nature, d'époques concernées, etc.), rendant compte d'une évolution de la conception du patrimoine. Aussi, ces deux processus ont connu une diffusion spatiale de l'Europe vers l'ensemble de l'Occident, pour ensuite devenir mondiale (Choay, 1992). Toutefois, alors que cette diffusion concerne la désignation de patrimoine dans son acception " européo-centrée », laquelle est largement portée par l'Unesco, des sociétés extra-européennes avaient déjà leur propre conception du patrimoine. La Chine en constitue un exemple particulièrement stimulant à interroger.

3 Au premier regard, la Chine, comme la France et l'Europe, cherche à mettre en valeur son patrimoine (Bonard, Felli, 2008) et développe le tourisme sur son territoire depuis quelques décennies (Taunay, 2009). Cette situation se retrouve, entre autres, dans le cas de quartiers historiques des centres-villes. Tant en Europe qu'en Chine, les documents officiels de planification et d'urbanisme évoquent la revitalisation des quartiers anciens, de même que l'accueil des visiteurs. Mais derrière des termes similaires, retrouve-t-on les mêmes réalités? Surtout, les rapports aux lieux ${ }^{3}$ de ces visiteurs (et plus largement l'ensemble des usagers) sont-ils les mêmes? Pour quelles raisons les fréquentent-ils? Ces lieux sont-ils recherchés pour leur caractère patrimonial ? Afin d'apporter des éléments de réponse à ces questions, nous avons pris pour analyse un type de lieux souvent occulté par les chercheurs non chinois, soit les quartiers anciens de villes chinoises qui attirent peu de visiteurs occidentaux et ne sont que partiellement soumises à l'influence occidentale, contrairement aux grandes métropoles ou encore aux villes inscrites sur la Liste du patrimoine mondial. Plus exactement, c'est un quartier ancien de la ville portuaire de Ningbo, NanTangLaoJie, qui a servi de cas d'étude. Nous avons cherché, d'une part, à cerner les pratiques des usagers de ce quartier mis en tourisme et patrimonialisé de même que leurs motivations et, d'autre part, à comprendre ce qui « fait patrimoine » pour ces individus.

4 Le propos s'articulera en trois temps. Nous effectuerons d'abord un retour sur les différentes conceptions de la notion de patrimoine, de l'Europe à la Chine, avant de nous intéresser plus spécifiquement aux quartiers urbains centraux anciens dédiés au tourisme et aux loisirs. Une seconde partie portera sur l'enquête de terrain, avec une présentation de Ningbo et du quartier NanTangLaoJie dans un premier temps, puis en évoquant la méthode d'enquête et les contraintes inhérentes au travail de terrain en Chine. En troisième et dernière partie de l'article, seront abordés les principaux éléments de résultats, à travers les représentations et pratiques des usagers de NanTangLaojie.

\section{Création des quartiers patrimoniaux et touristiques : du modèle occidental à la version chinoise}

\section{Patrimoine : une notion singulière, des acceptions plurielles}

5 Le patrimoine, tel que mobilisé aujourd'hui par des instances internationales comme l'Unesco, est une création européenne. De l'intérêt pour les ruines antiques à la Renaissance à celui pour les monuments historiques ( $18^{\mathrm{e}}$ et $19^{\mathrm{e}}$ siècles), il connaît une 
mutation majeure au cours du $20^{\mathrm{e}}$ siècle. D'une part, il ne porte plus uniquement sur le bâti, incluant désormais la nature et l'immatériel, d'autre part il ne se limite plus à des biens isolés, en intégrant par exemple des quartiers anciens entiers (Choay, 1992 ; Petti et al., 2019). En plus de ces extensions typologique et chronologique, il est possible d'identifier une extension géographique, le patrimoine tendant à devenir mondial et universel, entre autres à travers la Convention du patrimoine mondial portée par l'Unesco, établie en 1972 (Choay, 1992). L'apparition de ces différentes formes d'extensions patrimoniales est consubstantielle à la considération d'un patrimoine « construit » et non « donné » (Leniaud, 1992; Carman, 2002). Il est ainsi aujourd'hui admis que le patrimoine relève d'une construction sociale impliquant de facto des variations en matière de sens et d'acceptions d'une époque à une autre, mais aussi d'une société à une autre. En ce sens, l'intérêt de la question patrimoniale en contexte chinois repose particulièrement sur deux éléments: d'une part, l'ancienneté d'une notion semblable à l'européenne et d'autre part le décalage important du sens qu'elles recouvrent en Chine et en Europe.

6 En Chine, comme en Europe, le patrimoine a une histoire très ancienne, laquelle remonte aux dynasties du Sud (420-589) (Zhang, 2017). Les termes utilisés ainsi que le sens qu'ils recouvrent ont connu des évolutions importantes, de yichan («biens laissés par les morts»), à lishi guji (renvoyant à l'idée de monuments historiques) jusqu'à wenhua yichan (" patrimoine culturel ») apparu en 1985 lors de l'adhésion du pays à la Convention du patrimoine mondial portée par l'Unesco. Comme en Europe, la volonté de préservation y est également corrélée à des destructions de biens patrimoniaux. La fin de la dynastie Qing (1644-1911), marquée par les guerres de l'Opium et des mouvements de révoltes internes, entraînant d'importantes destructions (et spoliations) de "reliques culturelles", voit ainsi émerger le premier inventaire du patrimoine au niveau national en 1909 (Zhang, 2017). Toutefois, et contrairement au contexte européen, l'ancienneté et l'authenticité n'y ont jamais eu une importance déterminante pour définir la valeur d'un bien patrimonial. Dans la tradition chinoise, la valeur tient davantage à ce que l'élément patrimonial représente, à la mémoire qui lui est associée, au fait qu'il incarne le chuantong ("tradition»). Par ailleurs, la réalisation de reproductions ou de copies y a longtemps été considérée comme une réponse réaliste à l'inévitable détérioration des biens au fil du temps (Chang, 1999). Si cette notion de patrimoine est polysémique et protéiforme, nous nous intéresserons ici à une seule de ses figures, soit les quartiers urbains anciens.

\section{Les quartiers urbains patrimoniaux et touristiques : mêmes mots, mêmes réalités?}

7 Les quartiers centraux anciens des villes européennes comptent parmi les espaces qui sont quasi systématiquement mis en valeur par les communes. Dans un contexte de « retours en ville » (Bidou-Zachariasen, 2003) des classes moyennes et supérieures, ces quartiers subissent des opérations de réhabilitation et de marketing visant à y attirer de nouveaux résidents, des touristes et, entre les deux, toute une gamme de visiteurs, allant des résidents des autres quartiers de la ville à ceux des communes voisines en passant par les voyageurs d'affaire.

8 Tandis que, jusqu'au début $\mathrm{du} 20^{\mathrm{e}}$ siècle, le patrimoine européen concernait essentiellement des monuments isolés, l'architecte et ingénieur italien G. Giovannoni 
est le premier à évoquer le concept de patrimoine urbain. Ses écrits ont directement influencé la première loi italienne sur les ensembles historiques (1939), mais leur influence se fait sentir également ailleurs en Europe. À titre d'exemple, la conception des secteurs sauvegardés définie dans le cadre de la loi française dite Loi Malraux (1962) est inspirée des travaux de Giovannoni (Choay, 2015 [1988]). Si le but de cette loi est de protéger le patrimoine que représentent ces ensembles bâtis, il y a volonté d'éviter d'en faire des musées à ciel ouvert. Il s'agit de maintenir un équilibre entre les travaux de rénovation nécessaires à l'habitabilité du bâti dans des conditions de confort et d'hygiène acceptables et la préservation des éléments considérés comme ayant un intérêt patrimonial. Ces secteurs, de même que d'autres quartiers anciens, constituent par ailleurs des attraits touristiques au cœur des villes, les touristes (majoritairement européens) étant particulièrement séduits par l'ambiance pittoresque et l'authenticité de ce bâti.

9 A première vue, les villes chinoises sont engagées dans une dynamique similaire. Depuis 1982, l'État, sur proposition du Ministère de la Construction et du Bureau du patrimoine, a établi trois listes de «Villes historiques et culturelles» (Lishi wenhua Mingcheng), totalisant près d'une centaine de villes (Guo, 2008). Chacune d'elle peut sélectionner des quartiers à valeur historique et leur appliquer des plans de protection spécifiques. Comme en Europe, cette émergence de la prise en compte du patrimoine urbain s'articule à la diffusion du tourisme dans une logique de renforcement de l'économie (Bi et al., 2015). Suite à l'arrivée au pouvoir de Deng Xiaoping, la Chine s'est urbanisée et modernisée, entraînant une nette amélioration du niveau de vie des habitants, tout particulièrement des citadins. L'émergence de cette nouvelle classe moyenne urbaine couplée à l'instauration des premiers congés payés à la fin des années 1990, a permis au tourisme et aux loisirs de se développer (Taunay, 2009 ; Bi et al., 2015). Aussi, le même engouement des touristes et autres visiteurs pour les sites et quartiers patrimoniaux semble présent en Chine et en Europe. Toutefois, les opérations menées dans ces quartiers tout comme les attentes des touristes chinois, sont, dans certains cas, diamétralement opposées aux situations observées en Europe. Plusieurs travaux conduits en Chine par des chercheurs occidentaux (Oakes, 1997; McKhann, 2001; Taunay, 2009) ont permis de mettre en lumière des différences très marquées entre les attentes des touristes occidentaux et chinois, ces derniers étant moins à la recherche de lieux authentiques que confortables, modernes et bien dotés en commerces. Les quartiers anciens mis en tourisme ne font pas exception. Même s'ils sont promus en tant que quartiers "historiques", les opérations qui y sont menées renvoient moins à une volonté de préservation du bâti qu'à une évocation de la mémoire des lieux. Plus concrètement, ces opérations de réhabilitation des quartiers prennent fréquemment la forme de "destruction constructive » $(\mathrm{Fu}, 2015, \mathrm{p} .124)$, soit le fait de « déloger ${ }^{4}$ les résidents (Zhang, 2013), démolir tout ou partie du bâti existant et de le reconstruire dans un style architectural rappelant la Chine impériale (Guo, 2008). 


\section{En quête ${ }^{5}$ 'un quartier urbain chinois mis en tourisme et en patrimoine : NanTangLaoJie}

\section{Mutation d'un quartier ancien habité en un lieu dédié aux loisirs et au tourisme}

NanTanLaojie est l'un des huit quartiers historiques de Ningbo. Cette ville portuaire, située à environ $200 \mathrm{~km}$ au Sud de Shanghai (Figure 1), a été inscrite sur la seconde liste nationale des «Villes historiques et culturelles " (Lishi wenhua Mingcheng) en 1986. Un "Plan de protection de la ville historique et culturelle de Ningbo", produit par le département d'urbanisme de la ville, régit les principes directeurs d'aménagement des huit quartiers historiques, en accord avec les règlementations nationales et provinciales.

Depuis les années 1990, la ville met en œuvre des opérations de revitalisation urbaine de ses quartiers anciens dans une dynamique de mise en tourisme (Zheng et al., 2015), lesquelles sont portées par la Ningbo City Investment Holdings Compagny. Aussi, seuls deux des huit quartiers historiques n'avaient pas encore été soumis à des opérations de réhabilitation complète à l'été 2018. Pourtant, Ningbo peut encore difficilement être considérée en tant que ville touristique. En reprenant les caractéristiques des lieux touristiques définies par les chercheurs de l'Équipe MIT (2002), Ningbo correspond plutôt à une ville-étape. En effet, la ville sert de point de départ pour explorer les sites touristiques des environs, dont l'île de Putuoshan (et plus largement l'ensemble de l'archipel de Zhoushan). Ces opérations de réhabilitation s'inscrivent ainsi dans une volonté de renforcement du caractère touristique de Ningbo, visant à stimuler ce secteur économique. 
Figure 1. Carte de localisation des quartiers historiques de Ningbo.

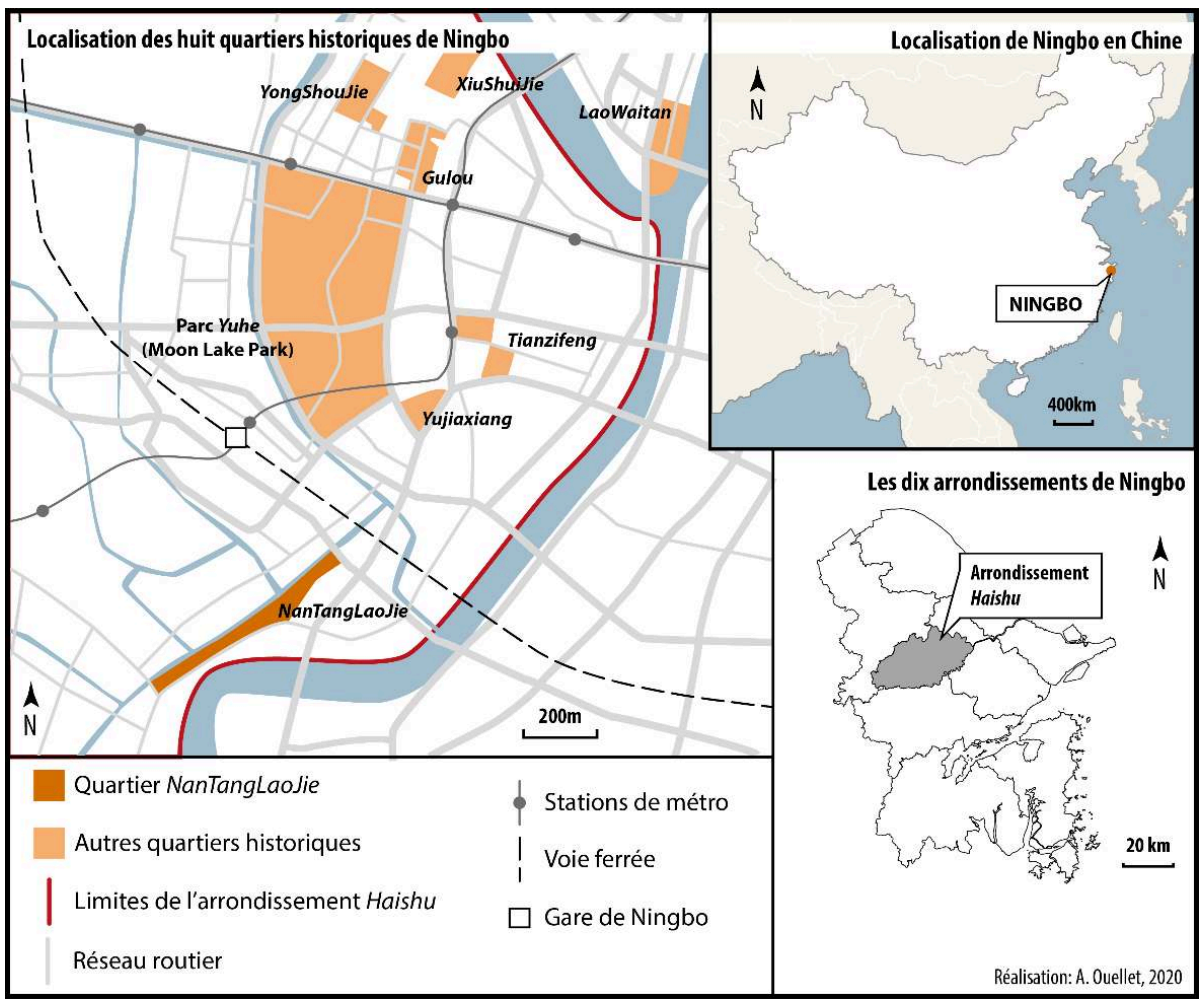

Il y a encore douze ans, le quartier de NanTangLaoJie, situé au cœur de l'arrondissement central de Haishu, présentait un bâti très dégradé, comme beaucoup de quartiers centraux historiques des villes chinoises. Le projet de réhabilitation du bâti et de mise en tourisme du lieu a été entrepris en 2009. Les résidents ont alors été délogés et une partie des bâtiments a été démolie et reconstruite selon un style architectural rappelant la Chine impériale (Chen et al., 2013). Ce chantier s'est organisé en 2 phases. La première phase, achevée en 2012, correspondait plutôt à une restauration ou réhabilitation du bâti, tandis que la seconde, complétée en 2015, a davantage relevé de la destruction-reconstruction. Selon les documents de planification et de protection des quartiers historiques de Ningbo, le but visé par la transformation du quartier était d'en faire une «zone commerciale traditionnelle qui intègre les monuments historiques, le tourisme, la culture et les loisirs $»^{7}$. Le quartier regroupe aujourd'hui essentiellement des commerces en lien avec la gastronomie (restaurants, vente à emporter, épicerie de produits locaux, etc.), mais aussi un hôtel, des librairies, des boutiques de souvenirs et d'artisanat, etc. Ses usagers comprennent des résidents de Ningbo, des commerçants y travaillant mais aussi des touristes et autres voyageurs. Quelques étrangers, dont des Occidentaux, font partie de ce dernier groupe, bien que les Chinois soient largement majoritaires ${ }^{8}$.

\section{Enquêter au sein d'un quartier d'une ville chinoise : méthodologie et contraintes}

13 Au niveau de la pratique de terrain et de la production de matériau, il est nécessaire d'effectuer un bref retour sur les contraintes inhérentes à tout terrain en contexte étranger et les conditions spécifiques du travail mené à Ningbo. 

d'enseignant-chercheur en géographie au sein de l'Institut conjoint des universités de Ningbo et Angers à Ningbo durant l'année universitaire 2017-2018. Tel qu'évoqué en introduction, le choix de Ningbo comme terrain d'enquête, bien qu'influencé par des raisons logistiques ${ }^{9}$, se justifie pleinement par le fait que la grande majorité des travaux menés en Chine sur la thématique des transformations des quartiers anciens (et diffusé hors de Chine) se concentre sur les métropoles dont Shanghai (Ged, 1996 ; Guo, 2008 ; Zhang, 2013), Pékin (Lefebvre, 2017) ou Tianjin (Debelle, 2015), ou encore les villes dont le patrimoine est inscrit sur la Liste du patrimoine mondial dont Lijiang (McKhann, 2001) ou Pingyao (Wang, 2011). Le cas de Ningbo permet de s'inscrire dans le prolongement de ces travaux en élargissant le spectre des villes concernées par ces transformations. des entretiens dans la langue locale, le mandarin. Pour pallier cet obstacle, deux principaux contournements ont été mis en place ${ }^{10}$, soit la pratique de l'observation et la réalisation d'entretiens directifs avec l'aide d'un tiers. D'abord, des séances d'observation menées systématiquement à différents moments de la journée, mais aussi en semaine et durant le week-end, ont permis de recueillir des informations concernant l'organisation du quartier, les usages et les usagers. L'observation permettant de voir ce que les gens font mais non de comprendre le sens donné à ces pratiques, des entretiens directifs ont ensuite été conduits avec l'aide d'étudiantes inscrites dans le cursus sino-français de l'Institut conjoint ${ }^{11}$. Un total de 80 entretiens a ainsi été réalisé en mai et juin 2018. Les usagers étaient abordés au sein du quartier, de manière aléatoire et sans prise de rendez-vous préalable. Trois catégories d'usagers du quartier sont concernées : les commerçants et employés travaillant dans le quartier ${ }^{12}$ (25), les résidents de Ningbo (32) et les touristes et voyageurs d'affaire (23) ${ }^{13}$. L'objectif des entretiens était de comprendre ce que les usagers viennent faire à NanTangLaoJie, pourquoi ils y viennent, ce qu'ils apprécient (ou non), quelles représentations ils en ont, est-ce qu'ils le considèrent comme un lieu patrimonial (ou non) et pourquoi. Le matériau discursif ainsi produit a permis de saisir les représentations des usagers et d'affiner l'analyse des pratiques.

\section{Pratiques, représentations et discours des usagers du quartier}

\section{Pratiques d'un quartier dédié au tourisme et aux loisirs}

16 Avant d'entamer les entretiens au sein du quartier, les séances d'observation ont permis d'identifier les pratiques dominantes, toutes catégories d'usagers confondues. Les individus présents à NanTangLaojie déambulent, prennent des photographies, font des achats, entre autres de nourriture ensuite consommée dans la rue (youzanzi ${ }^{14}$, chòudòufu ${ }^{15}$, etc.). Peu d'éléments permettent de distinguer les différents types d'usagers. Seule une place (marquant la séparation des secteurs A et B (figure 2)) au centre du quartier semble rassembler des habitués, dont les pratiques se démarquent des autres usagers. Ces habitués restent assis durant plusieurs heures (et ne font rien d'autre) et certains semblent se connaître. 
L'analyse croisée du matériau discursif et des données issues de l'observation, permet à la fois d'affiner et de conforter ces premiers éléments et de constater que peu de distinctions se font jour entre les pratiques des résidents de Ningbo et celles des touristes ou autres voyageurs. La consommation, par l'achat de nourriture ou de souvenirs, la prise de photographies ${ }^{16}$, de même que la balade sont les activités prédominantes. Cette très forte similitude des pratiques peut s'expliquer, en partie, par deux éléments. D'abord, Ningbo n'étant pas réellement considérée comme une ville touristique; pour plusieurs touristes rencontrés, NanTangLaoJie n'est pas un site touristique à part entière mais simplement un lieu agréable où manger ou se balader entre les visites de sites touristiques plus réputés et situés à proximité (bibliothèque Tianyi, temple Baoguo, île de Putuoshan, etc.). Ensuite, les résidents du quartier ont été « délogés » (Zhang, 2013) avant la réhabilitation et la mise en tourisme du quartier. De fait, cette absence de résidents vivant sur place limite les pratiques relevant du quotidien (rencontrer des amis, faire des courses du quotidien, etc.). Seuls quelques enquêtés, vivant à proximité immédiate du quartier, y ont des pratiques de ce type. À titre d'exemple, une octogénaire habitant à proximité immédiate de NanTangLaoJie $\left(\mathrm{R} 32^{17}\right)$ explique y venir quotidiennement pour se balader et rencontrer ses amies. Par ailleurs, seuls des résidents de l'arrondissement Haishu ont affirmé fréquenter le quartier sur une base hebdomadaire (voire quotidienne).

Figure 2. Schéma du quartier NanTangLaoJie.

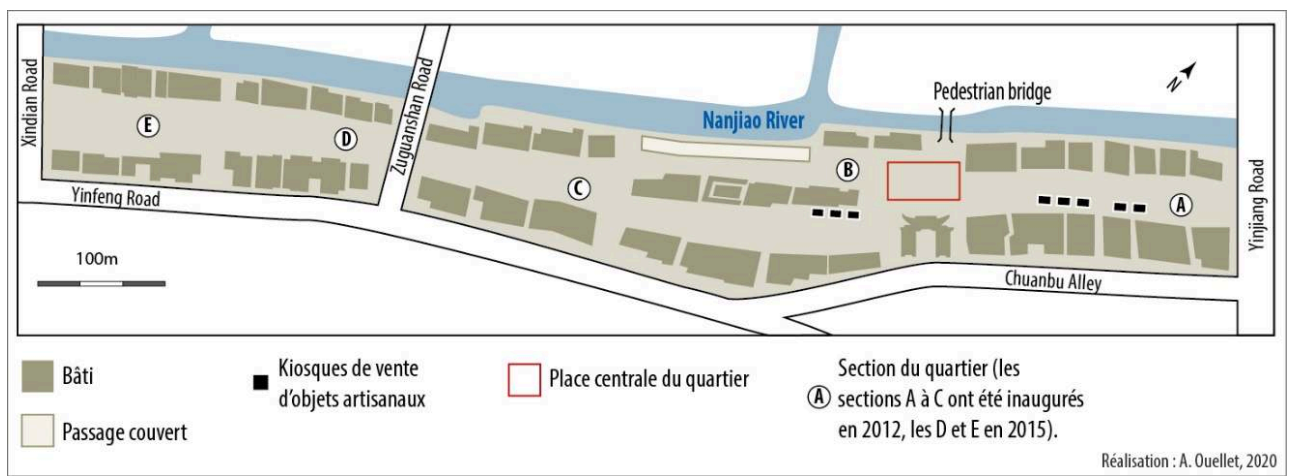

Figure 3. Des usagers du quartier font la queue pour acheter des youzanzi ; l'une des entrées du quartier, face à la place centrale (cf. figure 2 ).
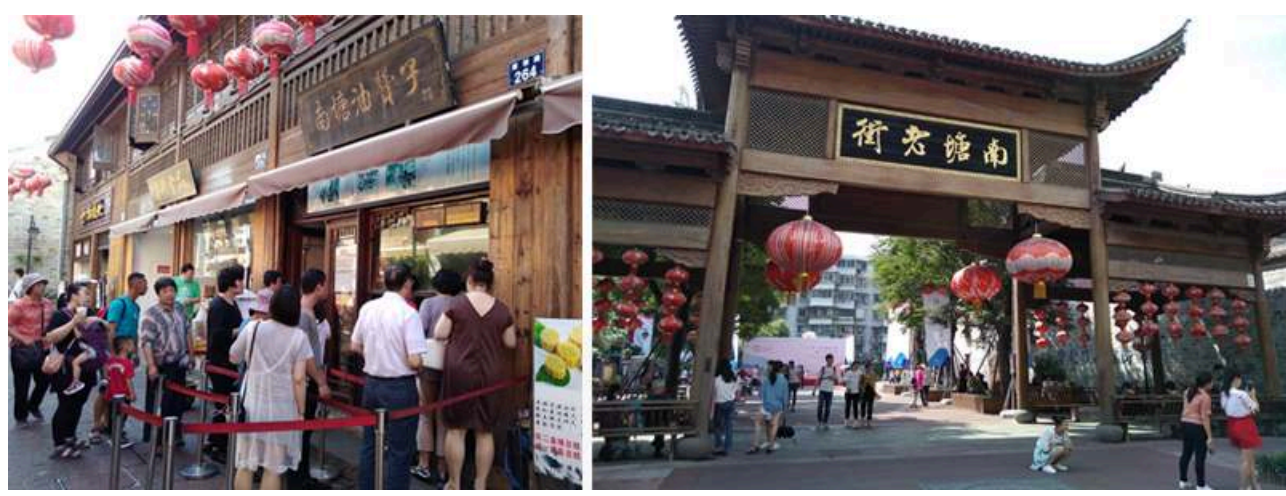


\section{Mise en perspective avec des travaux menés en France} touristiques françaises, la gamme de pratiques observées était beaucoup plus large (Ouellet, 2017). Nous postulons que cette situation s'explique par le fait que les centres anciens étudiés en France (Sarlat, Dinan) demeurent habités par des résidents permanents, tout en constituant une réelle destination touristique, le but premier de la visite de ces villes. Il était alors possible d'observer, dans un même lieu, des pratiques $\mathrm{du}$ quotidien comme aller faire ses courses, acheter son pain, tout autant que des pratiques « déroutinisantes» (Stock, 2005) comme la visite de monuments historiques. Ces extrêmes sont ainsi gommés dans le cas de NanTangLaojie entraînant donc un resserrement sur la partie centrale de cette échelle graduée des pratiques (Figure 4)

Figure 4. Échelle des pratiques. Mise en perspectives des situations observées dans de petites villes touristiques patrimoniales françaises et à NanTangLaoJie.

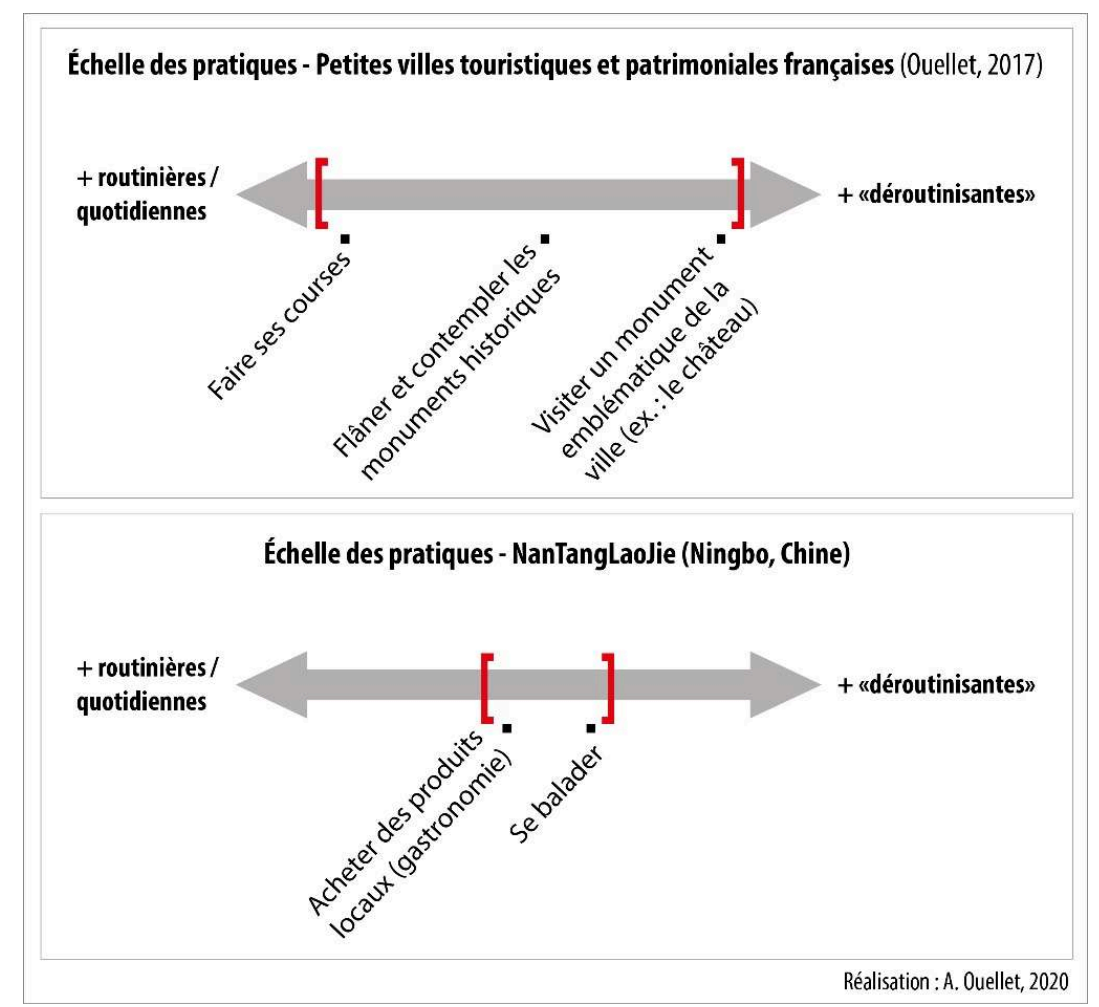

\section{Un quartier, des patrimoines}

L'une des thématiques abordées durant l'entretien concernait le patrimoine. Il était demandé à la personne enquêtée si elle considérait NanTangLaojie comme " historique » (lishi), comme un "patrimoine» (wenhua yichan) et pourquoi. Plus que la distinction entre les individus considérant que le quartier est patrimonial ou non, ce sont les raisons de ce choix qui nous intéressent ici.

L'analyse du matériau discursif recueilli permet de distinguer deux grandes catégories. Un premier groupe d'enquêtés a une conception du quartier historique qui renvoie à l'ancienneté et l'authenticité du bâti, se rapprochant ainsi de la vision "unescienne » du patrimoine. Pour ceux-là (29/80), le quartier n'est pas historique puisqu'en partie 
reconstruit. Ces enquêtés utilisent d'ailleurs un vocabulaire plus négatif pour le décrire, avec des termes tels que : " imitation ", «faux ", « déception » (respectivement 3, 2 et 1 occurrence.s). Si, chez les individus considérant que le quartier n'est pas patrimonial, l'argumentaire renvoie principalement au fait que le bâti a été reconstruit, il porte aussi, bien que de manière secondaire, sur l'aspect (trop) commercial du quartier.

«Ce n'est pas un quartier historique mais avant ça l'était. Maintenant, la

commercialisation supprime le caractère historique. $»^{18}$ (R07)

«Ce n'est pas vraiment un quartier patrimonial, plutôt commercial. Malgré que les

édifices aient l'air ancien, ils ont plutôt l'air d'avoir été refaits... » (V09)

L'aspect commercial est critiqué, semblant être antithétique avec le caractère historique du quartier. Le quartier, avec son bâti neuf de style ancien peut néanmoins en faire un lieu de balade agréable et servir de décor aux activités de loisirs et à la mise en scène de soi.

Toutefois, la majorité des enquêtés considère que NanTangLaoJie est un quartier patrimonial (51/80), avec une part de réponses positives beaucoup plus nette chez les résidents (24/32) que chez les voyageurs (12/23) ou les commerçants (15/25). Dans cette seconde catégorie, quatre conceptions se dégagent, bien que deux d'entre elles soient étroitement liées. Les plus nombreux sont ceux qui affirment que le quartier est patrimonial puisque le bâti est de style ancien (même s'il a été reconstruit), qu'il respecte le style architectural traditionnel. Le plus souvent un second argument vient s'ajouter à leur discours, soit le fait que la mémoire et l'histoire du lieu sont centrales, peu importe le bâti. S'opère, dans ce cas, un détachement entre la mémoire et valeur du lieu et le bâti en tant qu'enveloppe porteuse de cette valeur. Pour ces enquêtés, le style des bâtiments est traditionnel et évoque le passé, permettant de faire perdurer la mémoire du lieu.

"Même si une partie sont de nouveaux bâtiments, ils sont de style ancien. Ils

évoquent le passé, l'histoire du lieu. » (R31)

Une troisième conception, largement minoritaire, évoque une forme de patrimoine immatériel, à travers la culture locale, principalement les savoir-faire liés à la gastronomie. Cette vision est essentiellement portée par des résidents de Ningbo (3/32) et des commerçants (2/25) pour qui le quartier est patrimonial à la fois par son bâti de style ancien évoquant le passé et par les traditions culinaires qui y perdurent. Un restaurateur (C13) affirme ainsi que le quartier deviendra davantage patrimonial si ces savoir-faire sont mieux valorisés ${ }^{19}$. Enfin, il est possible de dégager un quatrième groupe parmi les répondants affirmant que le quartier est patrimonial, lesquels ne peuvent apporter de réels arguments, se limitant à dire que le quartier est « historique » puisque son nom l'indique (« Lao» signifiant « vieux»). La figure 5 résume ces différentes conceptions. 
Figure 5. Résumé des conceptions patrimoniales évoquées par les personnes enquêtées.

\begin{tabular}{|c|c|c|}
\hline $\begin{array}{l}\text { NanTangLaoJie, } \\
\text { un quartier patrimonial? }\end{array}$ & Principaux arguments mobilisés & $\begin{array}{l}\text { Nombre d'enquêtés concernés ( } R=\text { résidents; } \\
C=\text { commerçants } ; V=\text { voyageurs) }\end{array}$ \\
\hline $\begin{array}{l}\text { NanTangLaoJie n'est pas } \\
\text { un quartier patrimonial }\end{array}$ & $\begin{array}{l}\text { Le quartier n'est pas vraiment ancien } \\
\text { ou authentique, une grande partie } \\
\text { a été reconstruite }\end{array}$ & $\begin{array}{l}\text { 29/80, dont: } \\
\text { 8/32 (R);10/25(C);11/23(V) }\end{array}$ \\
\hline \multirow[t]{5}{*}{$\begin{array}{l}\text { NanTangLaofie est un } \\
\text { quartier patrimonial }\end{array}$} & $\begin{array}{l}\text { Le bâti est de style traditionnel, } \\
\text { respecte le style «ancien» (même si }\end{array}$ & $\begin{array}{l}\text { 40/80, dont: } \\
18 / 32(\mathrm{R}) ; 12 / 25(\mathrm{C}) ; 10 / 23(\mathrm{~V})\end{array}$ \\
\hline & \begin{tabular}{l|l} 
en partie reconstruit) & Ces de \\
\end{tabular} & $\begin{array}{l}\text { Ces deux arguments sont mobilisés } \\
\text { par les mêmes enquêtés }\end{array}$ \\
\hline & $\begin{array}{l}\text { Le quartier rappelle la mémoire } \\
\text { du lieu, I'histoire de Ningbo }\end{array}$ & \\
\hline & $\begin{array}{l}\text { Le quartier met en valeur la culture } \\
\text { locale (gastronomie) }\end{array}$ & $\begin{array}{l}\text { 5/80, dont: } \\
3 / 32(\mathrm{R}) ; 2 / 25(\mathrm{C}) ; 0 / 23(\mathrm{~V})\end{array}$ \\
\hline & $\begin{array}{l}\text { Pas de réels arguments, seulement } \\
\text { que le nom y fait référence ( }(\mathrm{aO})\end{array}$ & $\begin{array}{l}\text { 6/80, dont: } \\
3 / 32(\mathrm{R}) ; 1 / 25(\mathrm{C}) ; 2 / 23(\mathrm{~V})\end{array}$ \\
\hline
\end{tabular}

Réalisation: A. Ouellet, 2021

\section{Éloge de la propreté}

Si plusieurs éléments tirés du discours des enquêtés avaient pu être partiellement anticipés (différentes visions de ce qu'est le patrimoine, importance de la balade, de la prise de photographies, etc.), il en est un qui nous a initialement étonné : l'importance des références à la propreté dans le discours des enquêtés. La propreté du quartier est évoquée fréquemment durant les entretiens et ce terme revient à onze reprises lorsque nous demandons aux enquêtés de décrire NanTangLaoJie en trois mots. La propreté fait entre autres partie des aspects positifs du quartier et est particulièrement appréciée par les usagers.

Pour comprendre cette question de la propreté, il faut sortir de NanTangLaojie et le comparer à d'autres quartiers anciens n'ayant pas subi la même transformation. Parmi les huit quartiers historiques officiels que compte la ville, seuls deux n'étaient pas encore réhabilités au moment de notre recherche et un seul était encore habité par des résidents permanents: Yongshoujie. Ce quartier présente, comme NanTangLaojie avant les travaux de réhabilitation, des éléments de voirie et des bâtiments vétustes et dégradés (murs abîmés et sales, trous dans la chaussée, etc.) (Figure 6). 
Figure 6. Seul quartier historique de Ningbo non restauré et encore habité (à l'été 2018) : YongShouJie.
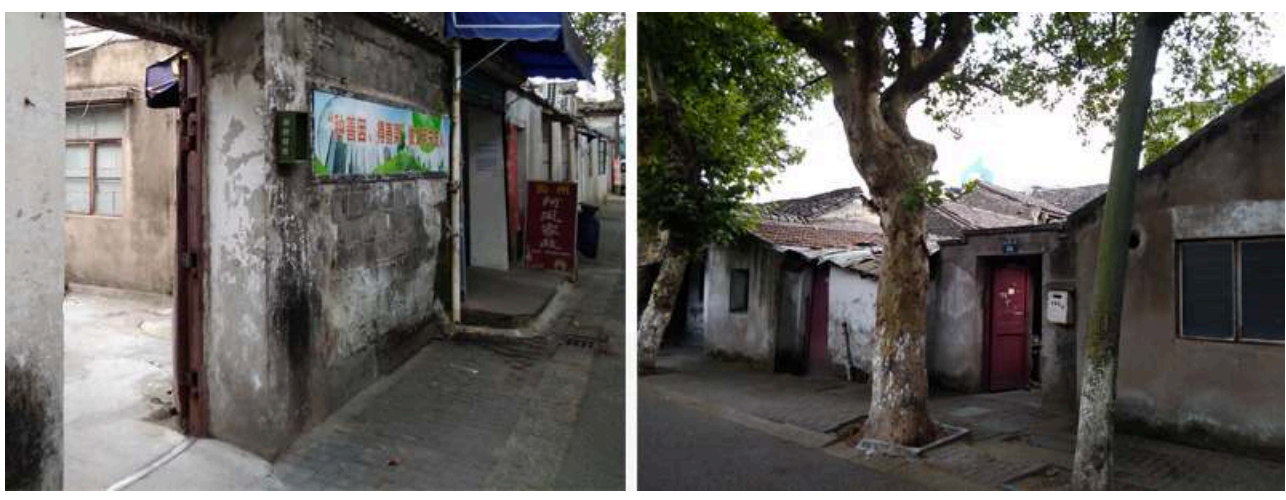

Aussi, lorsque les usagers étaient questionnés quant aux autres quartiers de Ningbo pouvant être qualifiés de patrimoniaux, aucun n'a évoqué les quartiers anciens non restaurés. Soit les enquêtés ne savaient que répondre ou ils nommaient systématiquement d'autres quartiers entièrement réhabilités tels que Gulou ou Yujiaxiang (Figure 7). Ces quartiers sont tous marqués par la présence de nombreux commerces, où les éléments bâtis de style ancien ont presque tous été reconstruits et font principalement office d'éléments de décor.

Figure 7. Exemples de quartiers entièrement restaurés (et dont le bâti a été en grande partie reconstruit) : Gulou (à gauche) et Yujiaxiang (à droite).
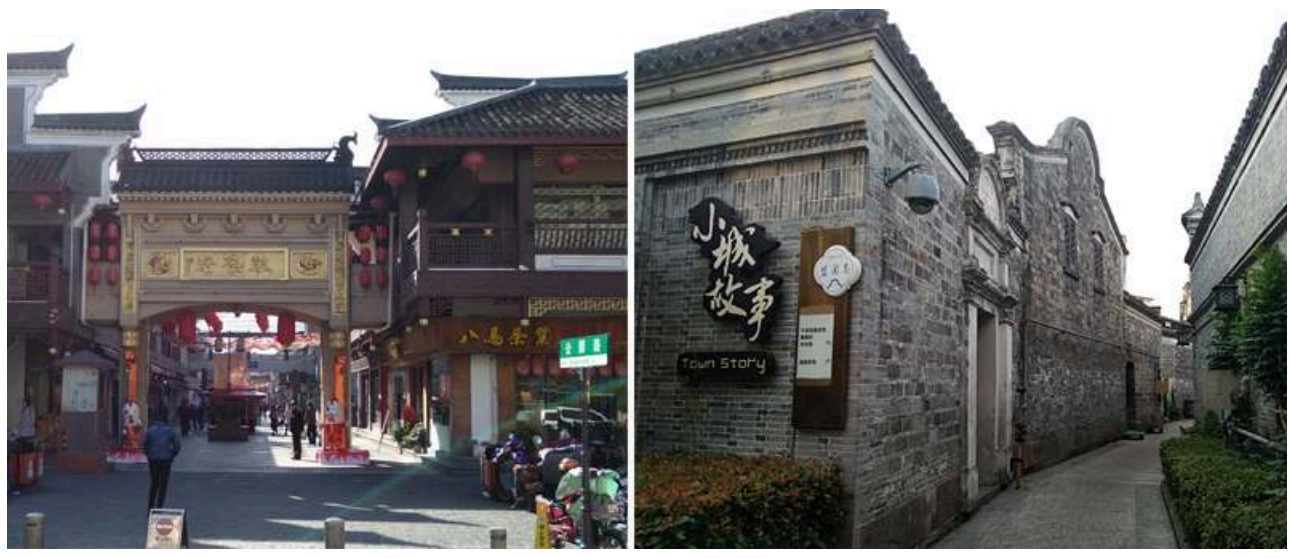

\section{Conclusion}

Au terme de cette étude, trois principaux constats peuvent être dégagés. D'abord, le fait que NanTangLaojie, comme beaucoup d'autres quartiers anciens en Chine ait été vidé de sa population résidante pour devenir un lieu commerçant, limite la variété des pratiques qui s'y déroulent. Cette situation se trouve accentuée par le fait que Ningbo constitue, pour le moment, davantage une ville-étape qu'une ville touristique. Ainsi, les touristes rencontrés à NanTangLaojie le considèrent davantage comme un lieu agréable où se balader ou se restaurer entre deux visites qu'en tant que véritable lieu touristique. Par ailleurs, la recherche menée dans ce quartier met en lumière un rapport au patrimoine et au passé qui se détache encore largement de celui qui prévaut en Europe. Tant les résidents que les touristes ou autres voyageurs fréquentant le 
quartier ne recherchent pas tant l'authenticité qu'un rappel de la mémoire du lieu, dans un cadre agréable et moderne. Même si certaines personnes rencontrées dans ce quartier, dont une majorité de touristes, ne le considèrent pas comme étant patrimonial puisqu'en grande partie reconstruit, la plupart des enquêtés sont peu gênés par cette reconstruction. Les quartiers où le bâti pourrait être considéré plus "authentique", au sens où il n'a pas encore subi de réhabilitation, ne sont jamais évoqués en tant que quartiers patrimoniaux. Le matériau discursif conforte ainsi les écrits de Tim Oakes (1997) quant à la primauté du confort et de la modernité (et nous ajouterions de la propreté) sur l'authenticité et l'ancienneté du patrimoine bâti. Enfin, bien que la situation observée à NanTangLaoJie se distingue grandement, au premier regard, de ce qui prévaut dans les quartiers centraux anciens européens, un aspect demeure constant, soit l'étroite relation entre la patrimonialisation et la transformation de lieux dédiés aux loisirs et au tourisme. Il s'agit dans les deux cas de réhabiliter ces quartiers de manière à satisfaire aux attentes de la majorité des visiteurs, que cela se fasse en valorisant l'authenticité ou la modernité. Néanmoins, le fait que Ningbo cherche à renforcer son attractivité touristique et que les voyageurs soient les usagers du quartier les plus critiques quant au manque d'authenticité amène à s'interroger sur le devenir des quartiers anciens qui n'ont pas encore subi d'opération de revitalisation. À titre d'exemple, le patrimoine urbain du quartier YongShoujie évoqué plus haut, conservait à l'été 2018 , son « état originel ", quoique dégradé, et était uniquement mis en valeur grâce à des plaques apposées sur les bâtiments. Tandis que le «Plan de protection du quartier historique et culturel YongShouJie " évoque la protection de l'authenticité du lieu et le maintien de la vie sociale, il semble y avoir une amorce de changement (ou à tout le moins une volonté de diversité) dans la manière d'aménager les quartiers historiques de la ville.

\section{BIBLIOGRAPHIE}

BI L., VANNESTE D. \& VAN DER BORG J. (2015), “Heritage Conservation, Urban Development and tourism in China since 1949: A regime Approach”, in GO F., AVAREZ M.D., VAN DER LAARSE R. \& EGBERTS L. (dir.), Proceedings 'Heritage, Tourism \& Hospitality' International Conference 2015, Amsterdam, CLUE+ Research Institute - Vrije Universiteit Amsterdam, pp. 33-45.

BIDOU-ZACHARIASEN C. (dir.) (2003), Retours en ville: des processus de " gentrification » urbaine aux politiques de « revitalisation » des centres, Paris, Descartes, $267 \mathrm{p}$.

BONARD Y., FELLI R. (2008), « Patrimoine et tourisme urbain. La valorisation de l'authenticité à Lyon et Pékin », Articulo - Journal of Urban Research, 4, http://articulo.revues.org/719

CARMAN J. (2002), Archaeology and Heritage: An Introduction, London, New York, Continuum, 228 p.

CHANG W.-C. (1999), «Esquisse d'une histoire du concept chinois de patrimoine », Publics et Musées, 15, pp. 81-118. 
CHEN M., WU Y. \& WU C. (2013), “Performance Of Commercial Renovation And Optimization of Historical Districts: Ningbo Case (历史街区商业化改造绩效评估与优化策略－以宁波三大历史 文化街区为例)”, Urbanist (Guihuashi), 10, pp. 86-96.

CHOAY F. (1992), L'allégorie du patrimoine, Paris, Seuil, 272 p.

CHOAY F. (2015 [1988]), « Patrimoine », in MERLIN P., CHOAY F., Dictionnaire de l'urbanisme et de l'aménagement, Paris, Presses Universitaires de France, pp. 535-538.

DEBELLE D. (2015), Les nouvelles dynamiques du tourisme et de la patrimonialisation en Chine :étude des anciennes concessions et du quartier français de Tianjin en particulier, Thèse de doctorat en géographie, Paris, Tianjin, Université Paris I-Sorbonne, Tianjin University, $492 \mathrm{p}$.

DUMAZEDIER J. (1972 [1962]), Vers une civilisation du loisir ?, Paris, Seuil, 309 p.

FU Y. (2015), 《宁波历史文化街区保护与更新存在的问题与对策研究》 (“Study on Problems and Countermeasures to Protect and Renovate Historical and Cultural Blocks in Ningbo"), Journal of Ningbo Radio and TV University, 13, 2, pp. 123-128.

GERMES M. (2008), « En quête d'un “terrain corporel”, jeux de regards dans et sur la rue », Communication au colloque "A travers l'espace de la méthode : les dimensions du terrain en géographie", Arras, 18-20 juin 2008, https://halshs.archives-ouvertes.fr/halshs-00358930

GED F. (1996), « Shanghai : du patrimoine identitaire au décor touristique. Le laboratoire de la nouvelle Chine », Les Annales de la recherche urbaine, 72, pp. 79-88.

GUO J. (2008), La transformation du tissu urbain des villes chinoises et la conservation du centre historique : cas du Vieux-Shanghai, Thèse de doctorat en aménagement, Montréal, Université de Montréal, $450 \mathrm{p}$.

LEFEBVRE A. (2017), « Pékin entre patrimoine et modernité : une mutation de la morphologie urbaine observée par satellite depuis 50 ans », Cybergeo : European Journal of Geography, http:// journals.openedition.org/cybergeo/28038

LENIAUD J.-M. (1992), L'utopie française. Essai sur le patrimoine, Paris, Mengès, 180 p.

MCKHANN C. (2001), « Tourisme de masse et identité sur les marches sino-tibétaines. Réflexions d'un observateur ", Revue Anthropologie et Sociétés, 25, 2, pp. 35-54.

MERLE T. (dir.) (2017), Les espaces du tourisme et des loisirs, Neuilly, Atlande, 288 p.

MICHEL B., OUELLET A. (2020), « Faire de la recherche en Chine. Contraintes et enseignements du terrain en contexte étranger ", Carnet de géographes, http://journals.openedition.org/cdg/5277

MIT (Équipe) (2002), Tourismes 1. Lieux communs, Paris, Belin, 319 p.

OAKES T. (1997), Tourism and Modernity in China, London, New York, Routledge, 272 p.

OUELLET A. (2017), Coprésence et rapports à l'espace dans les petites villes touristiques et patrimoniales. Lecture croisée de Dinan (Côtes-d'Armor) et Sarlat-la-Canéda (Dordogne), Thèse de doctorat en géographie, Angers, Université d'Angers, $418 \mathrm{p}$.

PETTI L., TRILLO C. \& MAKORE B.C.N. (2019), “Towards a Shared Understanding of the Concept of Heritage in the European Context”, Heritage, 2, pp. 2531-2544, https://doi.org/10.3390/ heritage 2030155

STOCK M. (2004), «L'habiter comme pratique des lieux géographiques », EspacesTemps.net, https://www.espacestemps.net/articles/habiter-comme-pratique-des-lieux-geographiques/ 
STOCK M. (2005), « Les sociétés à individus mobiles : vers un nouveau mode d'habiter? ", EspacesTemps.net, https://www.espacestemps.net/articles/societes-individus-mobiles/

TAUNAY B. (2009), Le tourisme intérieur chinois : approche géographique à partir de provinces du sudouest chinois, Thèse de doctorat en géographie, La Rochelle, Université de La Rochelle, $474 \mathrm{p}$.

WANG S.-Y. (2011), "In search of authenticity in historic cities in transformation: the case of Pingyao, China", Journal of Tourism and Cultural Change, 9, 1, pp. 18-30.

ZHANG C. (2017), « L'évolution historique de la notion de "patrimoine" en Chine », L'Information géographique, 81, 2, pp. 75-93.

ZHANG K. (2013), « « Qui » déloge "qui” dans les projets de rénovation de la vieille ville de Shanghai ?», Perspectives chinoises, 1, pp. 29-39.

ZHENG S., LI C. \& YANG J. (2015), "Texture Protection and Remolding of Historical Blocks in Southeast China under Tourism Economy (旅游经济下的中国东南㳂海历史街区肌理保护和重 塑)", L'architecture de Chongqin (Chongqin jiazhu), 14, 1, pp. 12-16.

\section{ANNEXES}

Tableau 1. Liste des personnes enquêtées de la catégorie « Commerçants et employés »

\begin{tabular}{|c|c|c|c|}
\hline $\begin{array}{l}\text { Code } \\
\text { utilisé } \\
\text { dans le } \\
\text { texte }\end{array}$ & $\begin{array}{l}\text { Statut } \\
\text { (Propriétaire (P)- } \\
\text { Employé (E)/ } \\
\text { Genre (H-F)/ } \\
\text { Tranche d'âge }\end{array}$ & Type de commerce & $\begin{array}{l}\text { Localisation du commerce } \\
\text { (secteurs Aà E) / Début de } \\
\text { ''activité (ouverture du } \\
\text { commerce ou embauche, } \\
\text { selon le statut) }\end{array}$ \\
\hline 001 & $\mathrm{P} / \mathrm{H} / 40-49$ & Épicerie de produits locaux & $A / 2012$ \\
\hline $\mathrm{CO2}$ & $E / F / 18-29$ & Restauration rapide & $\mathrm{A} / 2012$ \\
\hline $\mathrm{CO3}$ & $\mathrm{P} / \mathrm{H} / 30-39$ & Épicerie de produits locaux & $\mathrm{A} / 2017$ \\
\hline CO4 & $\mathrm{P} / \mathrm{H} / 40-49$ & Restaurant & $B / 2015$ \\
\hline $\cos$ & $E / F / 18-29$ & Boutique de vêtements traditionnels & $B / 2017$ \\
\hline C06 & $E / H / 18-29$ & Librairie & c/2017 \\
\hline c07 & $E / F / 30-39$ & Restaurant & A/ 2018 (janvier) \\
\hline $\mathrm{cos}$ & $E / F / 18-29$ & Épicerie de produits locaux & A/ 2018 (juin) \\
\hline $\cos$ & $\mathrm{P} / \mathrm{H} / 60-69$ & Artisanat (kiosque (cf. Figure 2)) & A/2017 \\
\hline C10 & $\mathrm{P} / \mathrm{H} / 30-39$ & $\begin{array}{l}\text { Boutique de bijoux et accessoires de } \\
\text { mode }\end{array}$ & A/ 2018 (mai) \\
\hline C11 & $E / H / 30-39$ & Épicerie de produits locaux & A/2016 \\
\hline C12 & $E / F / 18-29$ & Vente de boissons & $A / 2013$ \\
\hline C13 & $\mathrm{P} / \mathrm{H} / 50-59$ & $\begin{array}{l}\text { Vente de gâteaux de riz (au rdc) et } \\
\text { salle de restauration (à 'átage) }\end{array}$ & $A / 2016$ \\
\hline C14 & $E / H / 30-39$ & Hôtel & E / 2018 (avril) \\
\hline C15 & $P / H / 50.59$ & Boutique vente de thé & $E / 2017$ \\
\hline C16 & $\mathrm{P} / \mathrm{H} / 40-49$ & Poterie & $E / 2018$ (février) \\
\hline C17 & $E / F / 30-39$ & Spa & $E / 2017$ \\
\hline C18 & $\mathrm{P} / \mathrm{H} / 50.59$ & Manège pour enfants & D/2017 \\
\hline C19 & $E / F / 30-39$ & Bar-restaurant & D/2017 \\
\hline $\mathrm{C} 20$ & $E / F / 18-29$ & Salon de tatouages & $D / 2018$ (février) \\
\hline C21 & $E / F / 18-29$ & Bar-restaurant & $D / 2016$ \\
\hline $\mathrm{C22}$ & $E / H / 18-29$ & Glacier & D/2015 \\
\hline $\mathrm{C} 23$ & $E / F / 30-39$ & $\begin{array}{l}\text { Boutique de souvenirs et accessoires } \\
\text { de mode }\end{array}$ & $D / 2017$ \\
\hline C24 & $E / F / 18-29$ & Café-librairie & $c / 2015$ \\
\hline $\mathrm{C} 25$ & $E / F / 30-39$ & Objets tressés (kiosque (cf. Figure 2)) & B / 2018 (avril) \\
\hline
\end{tabular}

Tableau 2. Liste des personnes enquêtées de la catégorie « Résidents » 


\begin{tabular}{|c|c|c|c|c|}
\hline 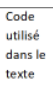 & $\begin{array}{l}\text { Lieu de résidence: } \\
\text { Arrondissement de Haishu; } \\
\text { Ningbo (autres } \\
\text { arrondissements) }\end{array}$ & $\begin{array}{l}\text { Genre (H } \\
-F) / \\
\text { Trinche } \\
\text { d'âge } \\
\text { d'âge }\end{array}$ & $\begin{array}{l}\text { Vit à Ningbo } \\
\text { depuis... }\end{array}$ & $\begin{array}{l}\text { Fréquence (approximative) de } \\
\text { frequentation de } \\
\text { Nantanglaodie }\end{array}$ \\
\hline R01 & Ningbo & $F / 18-29$ & Toute sa vie & Une fois par mois \\
\hline $\mathrm{R} 02$ & Haishu & $F / 18.29$ & 6 ans & 3.4 fois par an \\
\hline R03 & Haishu & $F / 30-39$ & 1 an & 3.4 fois par mois \\
\hline R04 & Ningbo & $F / 18-29$ & 6 mois & 1ere fois \\
\hline Ro5 & Ningbo & $H / 18-29$ & 1 an & $2^{\circ}$ fois \\
\hline R06 & Ningbo & $F / 30-39$ & 13 ans & 1-2 fois par an \\
\hline R07 & Haishu & $F / 18-29$ & Toute sa vie & 1-2 fois par mois \\
\hline R08 & Haishu & $H / 60.69$ & Toute sa vie & 1.2 fois par an \\
\hline RO9 & Haishu & $H / 18-29$ & 1 an & 1.2 fois par an \\
\hline $\mathrm{R} 10$ & Ningbo & H/30-39 & 9 ans & Moins d'une fois par an \\
\hline R11 & Ningbo & $H / 40-49$ & Toute sa vie & 1 fois par mois \\
\hline R12 & Ningbo & $F / 60-69$ & Toute sa vie & 1 fois par an \\
\hline R13 & Ningbo & $F / 18-29$ & 2 ans & 3 fois en 2 ans \\
\hline R14 & Haishu & $H / 30-39$ & 7 ans & 2 fois par semaine \\
\hline R15 & Ningbo & $H / 50.59$ & Toute sa vie & 1 fois par an \\
\hline R16 & Ningbo & $H / 30-39$ & Toute sa vie & $5-6$ fois par an \\
\hline R17 & Ningbo & $H / 18-29$ & 1 an & 1ere fois \\
\hline R18 & Ningbo & $H / 30-39$ & 1 an & $7-8$ fois par an \\
\hline R19 & Ningbo & $H / 18-29$ & Toute sa vie & 1ere fois \\
\hline R20 & Ningbo & $H / 40-49$ & 18 ans & $2^{\circ}$ fois \\
\hline R21 & Ningbo & $\mathrm{H} / 70$ et + & 50 ans & 1 fois par mois \\
\hline R22 & Haishu & $F / 70$ et + & Toute sa vie & 3.4 fois par mois \\
\hline R23 & Ningbo & $F / 18-29$ & Toute sa vie & 1ere fois \\
\hline R24 & Ningbo & $H / 30-39$ & 2 ans & 1ere fois \\
\hline R25 & Ningbo & $F / 18-29$ & 3 ans & 3 fois par an \\
\hline R26 & Ningbo & $H / 50-59$ & Toute sa vie & 4-5 fois par an \\
\hline R27 & Ningbo & $F / 30-39$ & Toute sa vie & 1 fois par an \\
\hline R28 & Haishu & $H / 60-69$ & Toute sa vie & 1-2 fols par semaine \\
\hline R29 & Ningbo & $F / 30-39$ & Toute sa vie & 1 fois par an \\
\hline R30 & Haishu & $\mathrm{H} / 70$ et + & Toute sa vie & 2 fois par mois \\
\hline R31 & Ningbo & $H / 60-69$ & Toute sa vie & 2-3 fois par mois \\
\hline R32 & Haishu & F/70 et * & Toute sa vie & Tous les jours \\
\hline
\end{tabular}

Tableau 3. Liste des personnes enquêtées de la catégories « Voyageurs »

\begin{tabular}{|l|l|l|l|}
\hline $\begin{array}{l}\text { Code } \\
\text { utilisé } \\
\text { dans le } \\
\text { texte }\end{array}$ & $\begin{array}{l}\text { Motif principal du séjour à Ningbo: Tourisme (T); } \\
\text { Voyage d'affaires (A) / Lieu de résidence habituelle } \\
\text { (province, municipalité ou ville (si Zhejiang)) }\end{array}$ & $\begin{array}{l}\text { Genre }(\mathrm{H}-\mathrm{F}) \text { / } \\
\text { Tranche d'âge }\end{array}$ & $\begin{array}{l}\text { Durée du } \\
\text { séjour } \\
\text { (nombre de } \\
\text { jours) }\end{array}$ \\
\hline V01 & T/ Shanghai & $\mathrm{F} / 60-69$ & 3 \\
\hline V02 & T/ Hainan & $\mathrm{F} / 18-29$ & 1 \\
\hline V03 & T/ Shandong & $\mathrm{H} / 30-39$ & 2 \\
\hline V04 & T/ Jiangxi & $\mathrm{F} / 50-59$ & 4 \\
\hline V05 & A/ Tianjin & $\mathrm{H} / 18-29$ & 1 \\
\hline V06 & T/ Shaanxi & $\mathrm{H} / 50-59$ & 2 \\
\hline V07 & T/ Shanghai & $\mathrm{F} / 60-69$ & 3 \\
\hline V08 & A/ Shaoxing & $\mathrm{F} / 18-29$ & 1 \\
\hline V09 & T/ Jiangxi & $\mathrm{F} / 30-39$ & 2 \\
\hline V10 & T/ Hangzhou (Zhejiang) & $\mathrm{F} / 18-29$ & 3 \\
\hline V11 & T/ Taizhou (Zhejiang) & $\mathrm{H} / 18-29$ & 2 \\
\hline V12 & T/ Wenzhou (Zhejiang) & $\mathrm{H} / 30-39$ & 1 \\
\hline V13 & T/ Shanghai & $\mathrm{F} / 60-69$ & 2 \\
\hline V14 & T/ Jilin & $\mathrm{H} / 40-49$ & 5 \\
\hline V15 & T/ Hangzhou (Zhejiang) & $\mathrm{H} / 18-29$ & 2 \\
\hline V16 & A / Hangzhou (Zhejiang) & $\mathrm{H} / 30-39$ & 1 \\
\hline V17 & T/ Shanghai & $\mathrm{H} / 60-69$ & 3 \\
\hline V18 & T/ Liaoning & $\mathrm{H} / 50-59$ & 2 \\
\hline V19 & T/ Liaoning & $\mathrm{F} / 30-39$ & 3 \\
\hline V20 & T/ Hunan & $\mathrm{H} / 30-39$ & 5 \\
\hline V21 & T/ Taizhou (Zhejiang) & $\mathrm{F} / 40-49$ & 2 \\
\hline V22 & T/ Shanghai & $\mathrm{F} / 40-49$ & 2 \\
\hline V23 & T/ Sichuan & $\mathrm{F} / 18-29$ & 4 \\
\hline & & & \\
\hline
\end{tabular}




\section{NOTES}

1. Nous considérons, dans la lignée des travaux de Mathis Stock (2004), que les résidents ne sont pas les seuls à habiter les lieux, l'habiter pouvant être plus ou moins temporaire.

2. Nous évitons de noter le terme patrimoine entre guillemets pour éviter d'alourdir le texte. Néanmoins, nous précisons que nous ne considérons pas sa définition comme étant stable et communément admise. En effet, nous cherchons justement à saisir le sens qu'il prend pour différentes sociétés et à questionner l'articulation entre le mot et les réalités qu'il désigne.

3. Nous considérons que le rapport aux lieux désigne à la fois les représentations de l'espace et les pratiques qui y sont réalisées.

4. Nous utilisons le terme « délogés » tel que mobilisé par Kai Zhang dans ses travaux portant sur le délogement des populations de la vieille ville de Shanghai. Ce délogement des résidents se fait généralement en échange d'un logement neuf en périphérie de la ville ou d'une compensation financière.

5. Nous reprenons ici le jeu de mots « en quête " (enquête), croisant les actions d'enquêter et de partir en quête de quelque chose, employé entre autres par Mélina Germes (2008).

6. L'île de Putuoshan est surtout célèbre pour la présence du mont Putuo, l'une des montagnes sacrées du bouddhisme en Chine.

7. Extrait du document: "Níngbō shì lìshǐ wénhuà míngchéng bǎohù guīhuà » («Plan de protection de la ville historique et culturelle de Ningbo », traduction personnelle), consulté le 22 juin 2018.

8. Ces éléments reposent sur le travail d'observation conduit sur place en 2017-2018.

9. Notre séjour en Chine étant articulé aux semestres de cours, le travail de terrain a dû être fait en parallèle des enseignements et devait donc être réalisé à Ningbo ou à proximité.

10. Pour des informations plus complètes et détaillées concernant la pratique du terrain, voir Michel B., Ouellet A. (2020).

11. Institut conjoint des universités de Ningbo et d'Angers à Ningbo.

12. Leur commerce ou emploi dans le quartier correspond, pour tous les enquêtés de ce groupe, à leur activité et source de revenu principales.

13. Une dizaine de personnes sollicitées ont par ailleurs refusé d'être interrogées. Ces refus ne concernaient pas un groupe en particulier en matière d'âge ou de genre. Leur refus ne nous permet pas de savoir à quelle catégorie appartenaient ces individus (résidents, touristes, etc.).

14. Sorte de crackers, pouvant être sucrés ou salés.

15. Sorte de tofu généralement traduit en anglais par «smelly tofu » ou «stinky tofu» (« tofu puant » en français).

16. D'après nos observations, il s'agit le plus souvent de photographies de soi (selfies). Les individus se mettent en scène dans le lieu, ce dernier servant à une valorisation de soi.

17. Les codes alphanumériques débutant par « $R$ » (résidents), " $C$ » (commerçants) ou « $V$ » (voyageurs) renvoient aux personnes enquêtées. La section « Annexes» en fin de texte, regroupe trois tableaux identifiant succinctement les 80 personnes rencontrées.

18. Tous les extraits d'entretien ont été traduits du mandarin vers le français avec l'aide de Liang Min, Liu Jingru, Xirou Yiang et Xu Linying, que nous tenons à remercier pour leur aide précieuse. 19. Si les entretiens auprès des commerçants ont un réel intérêt, ces derniers étant présents quotidiennement dans le quartier et ayant une connaissance fine de son fonctionnement, il faut néanmoins pour cette catégorie, être conscient que les aspects économiques peuvent influencer leur discours. 


\section{RÉSUMÉS}

Cet article explore la question des transformations des quartiers anciens en Chine. La contribution s'intéresse aux pratiques et représentations des usagers de NanTangLaoJie, un quartier de la ville de Ningbo, située sur la côte est chinoise. Ce quartier est emblématique de la situation de nombreux quartiers anciens du pays, soit un secteur dont ont été délogés les résidents permanents, qui a été entièrement restauré, voire reconstruit et qui est aujourd'hui dédié au tourisme et aux loisirs. Des entretiens directifs menés auprès des usagers du quartier permettent de mettre en avant des pratiques similaires (se balader, photographier, manger, faire du shopping) peu importe la catégorie d'individus concernés. Le matériau discursif permet aussi de dégager des représentations du patrimoine contrastées.

This paper explores the question of the transformations of old districts in China. The contribution focuses on the practices and representations of users of NanTangLaojie, a district of Ningbo, a city located on the east coast of China. This district is emblematic of the situation of many old Chinese districts: an area from which the permanent residents have been evicted, which has been completely restored, even rebuilt and which is now dedicated to tourism and leisure. Structured interviews conducted with users of the district allow to highlight similar practices (walking around, taking pictures, eating, shopping) regardless of the category of individuals involved. Interviews also make it possible to identify contrasting representations of heritage.

\section{INDEX}

Keywords : leisure and tourism, heritage, old districts, representations, practices, China

Mots-clés : loisirs et tourisme, patrimoine, quartiers anciens, représentations, pratiques, Chine

\section{AUTEUR}

\section{ANNIE OUELLET}

Docteure en géographie et chercheure associée à l'UMR CNRS 6590 ESO-Angers, annieouellet22@gmail.com 